\title{
Implementation of Dynamic Faces Based on Proxemics for Robot-ASD Children Interaction *
}

\author{
Giancarlo Pedroni Del Piero* Allan Deangelle Santos Mota** \\ Carlos Torturella Valadão*** \\ Eliete Maria de Oliveira Caldeira ${ }^{* * * *}$ \\ Teodiano Freire Bastos-Filho ${ }^{\dagger}$
* Postgraduate Program in Electrical Engineering, Federal University of Espirito Santo,ES, (e-mail: giancarlo.piero@aluno.ufes.br).
** Department of Electrical Engineering, Federal University of Espirito
Santo,ES, (e-mail: allan@vidadesilicio.com.br).
*** Postgraduate Program in Electrical Engineering, Federal University of Espirito Santo, ES, (e-mail: carlostvaladao@gmail.com).
**** Department of Electrical Engineering, Federal University of Espirito Santo, ES, (e-mail: eliete.caldeira@ufes.br).
$\dagger$ Postgraduate Program in Electrical Engineering, Federal University of Espirito Santo,ES, (e-mail: teodiano.bastos@ufes.br).

\begin{abstract}
This paper shows how a robot can be used to simulate facial expressions and, thus, increase the interaction between it and autistic children. These facial expressions are based on proxemics which uses information taken from a laser sensor. Following a proposed algorithm, the robot can choose an adequate face animation, expressing emotions in several degrees. The capability of displaying emotion that changes throughout the time with different intensities enhances the interaction between autistic children and socially affective robots, which can be applied as a support tool in their therapy. Moreover, once the robot acquires the proxemic information, it can change its behavior in an appropriate way to try catching the child's attention and interest. In this context, as both movement and facial expressions play an important role in this interaction, this way of processing emotion and displaying dynamic faces can bring a more natural and improved interaction.
\end{abstract}

Keywords: Autism, Mobile Robots, Social, Emotions, Dynamic Faces, Animation.

\section{INTRODUCTION}

According to Copeland (2018), autistic children have three main characteristics:

- Social interaction and communication problems;

- Difficulty relating to people, things and events; and

- Restricted and repetitive patterns of behaviors, interests or activities.

Additionally, The National Institute of Mental Health (2018) defines Autism spectrum disorder (ASD) as a developmental disorder that affects communication and behavior. Although autism can be diagnosed at any age, it is said to be a "developmental disorder" because symptoms generally appear in the first two years of life.

This work intends to improve autistic children social interaction using an assistive robot. For that, an assistive robot made at UFES, called New Mobile Autonomous Robot for Interaction with Autistics (N-MARIA) (Binotte, 2018) was developed at Federal University of Espirito Santo (UFES) to motivate ASD children to accept a closer

\footnotetext{
* Authors would like to acknowledge the financial support from the Fundação de Amparo à Pesquisa do Espírito Santo (FAPES).
}

contact to a robot, on proxemics zones defined as intimate and close intimate (see Section 1.1).

\subsection{Proxemics}

Proxemics is one of five major areas in nonverbal communication (Harper et al., 1978). Rios-Martinez et al. (2015) define as the study of spatial distances individuals maintain in various social and interpersonal situations.

About spatial distances, there are five concentric spatial zones affecting behavior, see Fig. 1 (Lambert, 2004):

(1) Close intimate zone;

(2) Intimate zone;

(3) Personal zone;

(4) Social zone;

(5) Public zone.

Studies such as Binotte (2018) show how proxemics can be used in a child-robot interaction. Following the concept that different interaction zones should have different robot behaviors, proxemics is a valuable way to select the appropriate robot action. Furthermore, expressions can also be modulated considering the children's actions and be- 


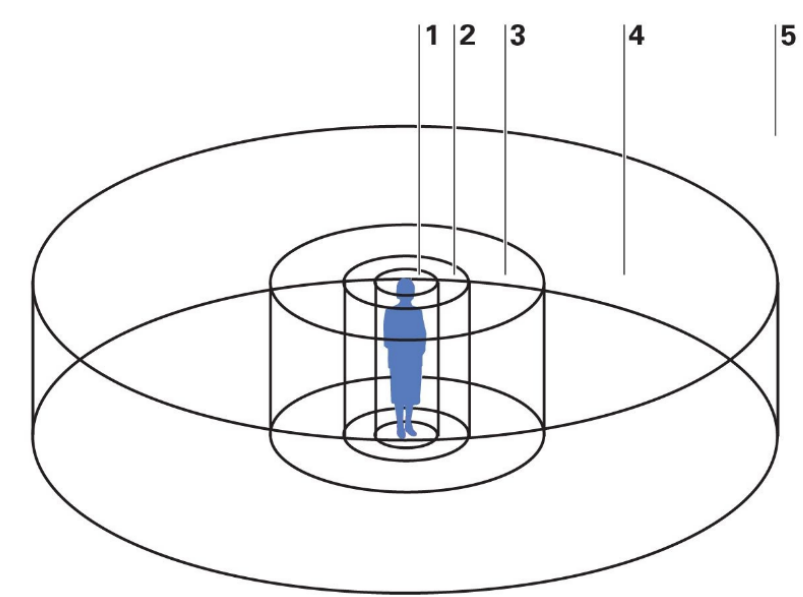

Figure 1. Spatial zones (Lambert, 2004).

haviors based on proxemics. Thus, some modulated faces can be used as response to a child-robot interaction, and proxemics can be used to do such modulations. Therefore, the idea consists of using some facial features to mimic different emotions and their intensity.

\subsection{Facial Action Coding System}

There are six main universal emotions: anger, disgust, fear, happiness, sadness, and surprise (Lafrance, 1979).

Representing these emotions, humans use many muscles of the face. Hjortsjö (1969) presented a first system to taxonomize human facial movements. This system was adopted by Friesen and Ekman (1978) and enhanced by Freitas-Magalhães (2018) and it is known as Facial Action Coding System - (FACS).

This paper presents an algorithm using action units to represent the six aforementioned expressions. To view some of these action units, please access Noldus Information Technology (2018) website.

The paper is organized as follows: in the first section, Introduction, the main aspects of the project, including proxemics and FACS, are explained; following, there is the Materials and Method section, where information about the robot and the system are given; next, in Results and in Discussion, are shown the expected results and the possible outcomes from it, respectively and, finally, the paper ends with the section Conclusions, which shows important aspects of working with emotion display in a child-robot interaction and the importance of this work for future applications.

\section{MATERIALS AND METHOD}

\subsection{Materials}

The robot N-MARIA is a $1.41 \mathrm{~m}$ tall toy-like robot, with both human and machine features, built with soft materials, such as Ethylene-vinyl acetate (EVA) painted in grey color. It has mobility features, given by a Pioneer 3-DX robot, located at the robot's base, together with the Light Detection And Ranging (LiDAR) laser device (SlamTec A2), and a 60 Ah lead-acid battery.

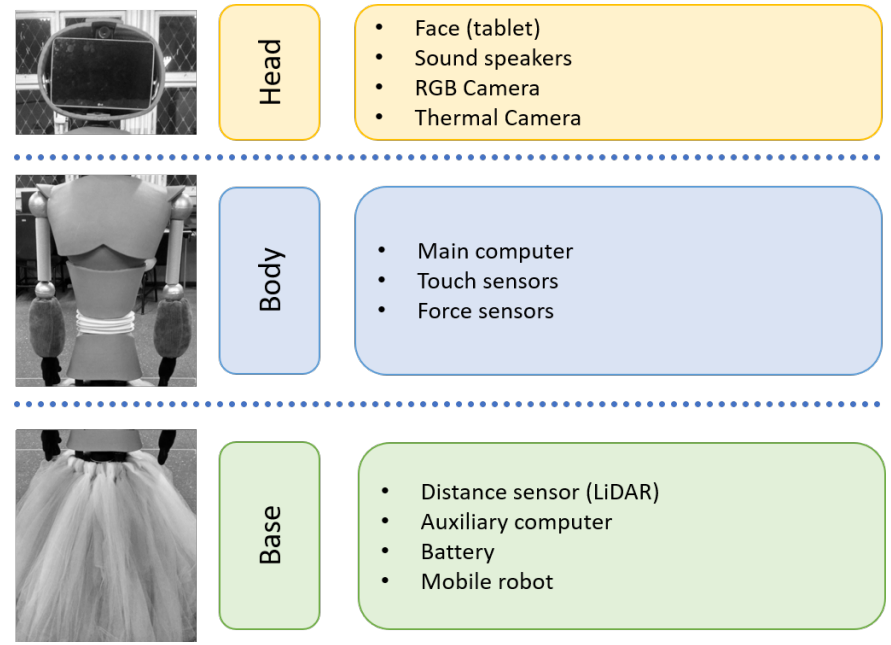

Figure 2. Hardware distribution inside N-MARIA's robot.

The base also contains an "auxiliary computer", which is one of the two NUCs installed in the robot. This computer is responsible for gathering the LiDAR data and send distance information to the "main computer" (the other NUC is installed in the middle part of the robot), and for sending the control commands to the Pioneer 3-DX driver. Figure 2 shows how these devices are placed in the robot structure.

The middle part of the robot contains capacitive touch sensors and resistive force sensors, along with the aforementioned "main computer", which runs a local web server for communication between devices. This computer collects information about the robot sensors and it is used by the control system to define the robot behavior. Therefore, there is a division of tasks between the two NUCs, being the "main computer" the one that processes the information about the interaction, control actions and system integration. On the other hand, the "auxiliary computer" uses the commands given by the "main computer" to apply the control laws and send the movement commands to the robot driver.

In the head, there are two cameras, which collect thermal and color image from children. These images are used by the robot to infer the child's emotion and to promote a better interaction by modulating the robot's behavior. Along with the cameras, sound speakers and a tablet are used to display speak programmed dialogues and affective face animation, respectively. These devices are controlled directly by the "main computer".

The robot was designed to bring the children's attention mainly to the head, where the emotions and expressions are displayed. Due to this reason, the body is mainly gray, with only some metal golden parts, which are touch sensors. A skirt is used in the base to hide the mobile robot and some electronic devices, which can deviate the children's attention. A picture of the robot, showing the structure and the embedded electronics in the base, is shown in Figure 3.

The tablet (facial expression) is one of the key points of the robot, which is useful to establish a friendly interaction. In order to present better facial expressions, animations that represent the intensity of emotion are used. Thus, it is 


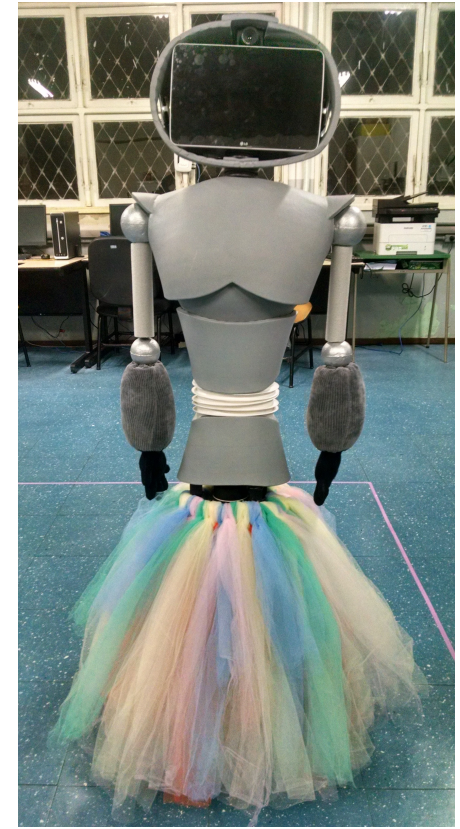

Figure 3. Picture of the robot N-MARIA. The mobility is possible due to the Pioneer 3-DX robot (located in the base).

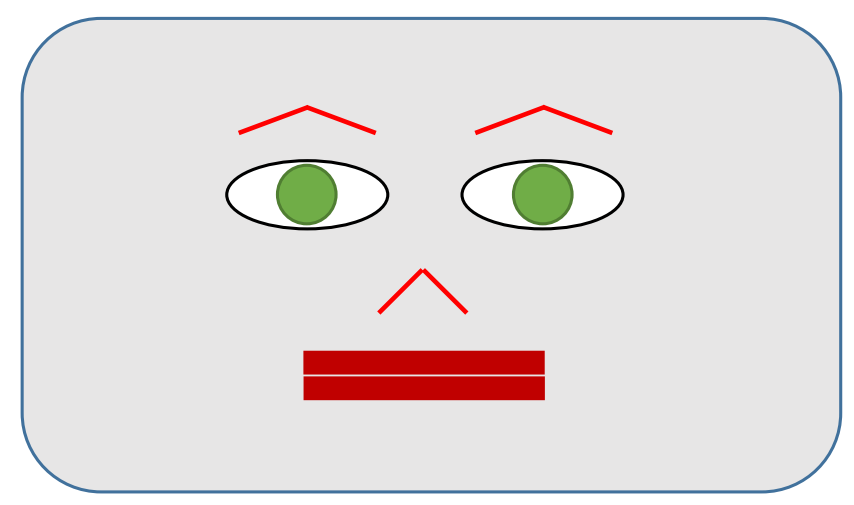

Figure 4. Proposed face.

possible for the robot to emulate the increase or decrease of an emotion towards the neutral state. More details can be viewed in Section 2.2.

\subsection{Method}

To express the intensity of its emotions in the display, the robot face uses six drawn objects. Figure 4 shows two brows, two eyes, one nose and one mouth, which are individual and can move independently.

Each face part has movements that emulate action units. A set of action units creates a face emotion, such as happiness or sadness.

Brow This object is used to represent two brows in the face. To create this object a rectangle is used to define the position, and within that rectangle two lines are drawn, one for the inner part of the eyebrow and other for the outer part. Thus, it is possible to control three action units: $\mathrm{AU}-01$, inner brow raiser; $\mathrm{AU}-02$, outer brow raiser; and AU-04, brow lowerer. See Figure 5.

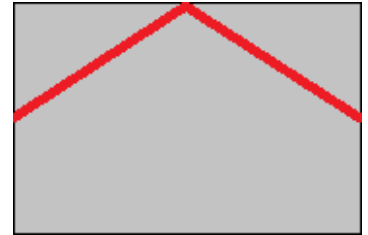

(a) Normal

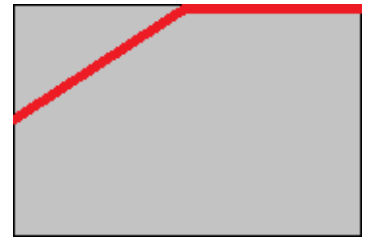

(c) $\mathrm{AU}-02$

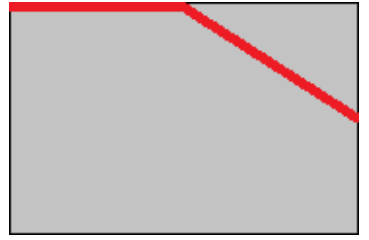

(b) $\mathrm{AU}-01$

(d) AU-04

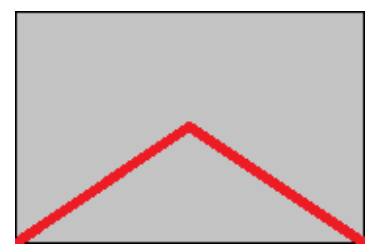

Figure 5. Action units that change brow object.

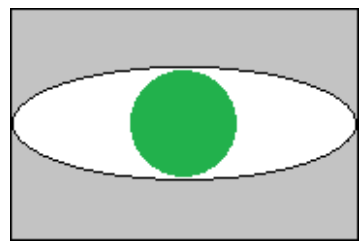

(a) Normal

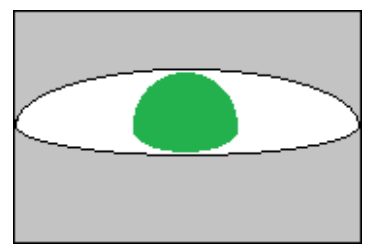

(c) $\mathrm{AU}-06$

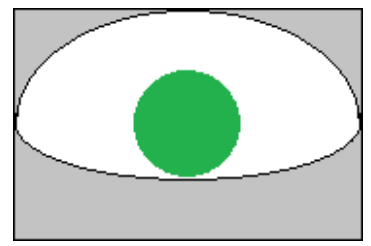

(b) $\mathrm{AU}-05$

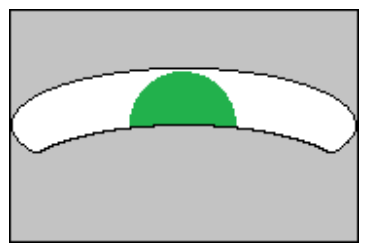

(d) $\mathrm{AU}-07$
Figure 6. Action units that change eye object.

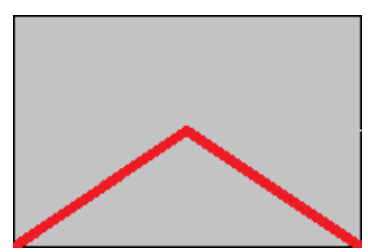

(a) Normal

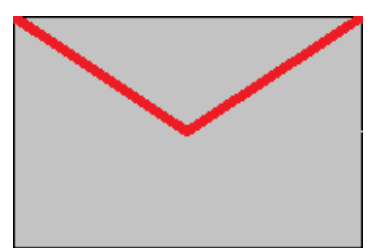

(b) AU-05
Figure 7. Action units that change nose object.

Eye This object is used to represent two eyes in the face. Like the brow, a rectangle defines the position of the eyes and inside this rectangle, the representation of iris, white and lid, is drawn using ellipses and arcs. So, this setup can form three action units: AU-05, upper lid raiser; AU-06, cheek raiser; and AU-07, lid tightener. See Figure 6.

Nose It is used to represent a nose in the face, and can show one action unit: AU-09, nose wrinkler. See Figure 7.

Mouth It is used to represent a mouth in the face. There are six possible action units: AU-12, lip corner pull; AU-15, lip corner depressor; AU-16, lower depressor; AU-20, lip stretcher; AU-23, lip tightener; and AU-26, jaw drop. See Figure 8. 


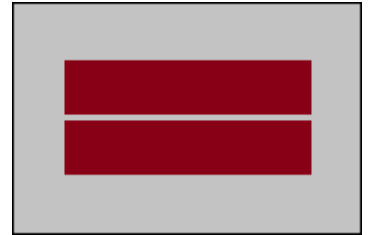

(a) Normal

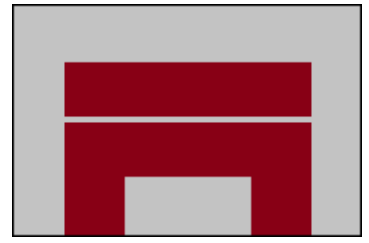

(c) $\mathrm{AU}-15$

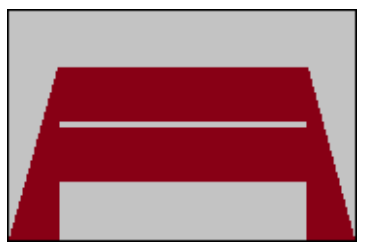

(e) $\mathrm{AU}-20$

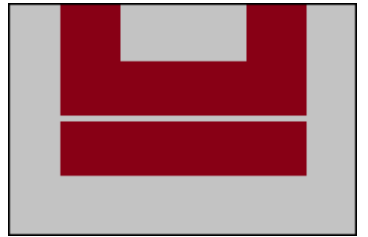

(b) $\mathrm{AU}-12$

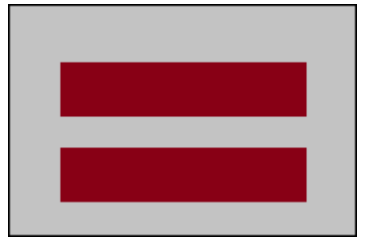

(d) $\mathrm{AU}-16$

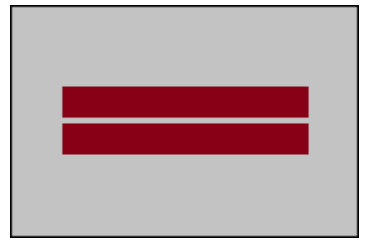

(f) $\mathrm{AU}-23$

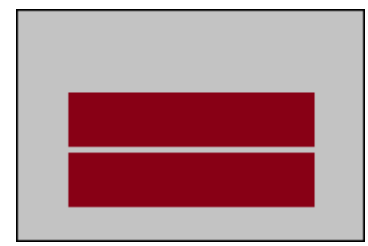

(g) AU-26

Figure 8. Action units that change mouth object.

\subsection{Factory of Emotions}

With the combination of some of the thirteen action units, it is possible to emulate all the six main universal expressions defined in Section 1.2.

The thirteen action units are:

- AU-01 inner brow raiser;

- AU-02 outer brow raiser;

- $\mathrm{AU}-04$ brow lowerer;

- AU-05 upper lid raiser;

- AU-06 cheek raiser;

- AU-07 lid tightener;

- AU-09 nose wrinkler;

- AU-12 lip corner puller;

- AU-15 lip corner depressor;

- AU-16 lower lip depressor;

- $\mathrm{AU}-20$ lip stretcher;

- AU-23 lip tightener;

- AU-26 jaw drop.

Table 1 lists which actions are used to emulate emotions. Figures $9 \mathrm{a}, 9 \mathrm{~b}, 9 \mathrm{c}$ and $9 \mathrm{~d}$ show how the action units form four expressions.

Facial emotions will change in response of theoretical model of Pleasure, Arousal and Dominance (PAD) (Bakker et al., 2014) and using mode Stimuli, Organism and Response (SOR) (Mehrabian and Russell, 1974).
Table 1. Emotions and its action units.

\begin{tabular}{ll} 
Emotion & Action Units \\
\hline Happiness & AU-06, AU-12, AU-26 \\
Sadness & AU-01, AU-04, AU-15 \\
Surprise & $\mathrm{AU}-01, \mathrm{AU}-02, \mathrm{AU}-05, \mathrm{AU}-16$ \\
Anger & $\mathrm{AU}-04, \mathrm{AU}-05, \mathrm{AU}-07, \mathrm{AU}-23$ \\
Disgust & $\mathrm{AU}-09, \mathrm{AU}-15, \mathrm{AU}-16$ \\
Fear & $\mathrm{AU}-01, \mathrm{AU}-02, \mathrm{AU}-04, \mathrm{AU}-05, \mathrm{AU}-07, \mathrm{AU}-20, \mathrm{AU}-26$ \\
\hline
\end{tabular}

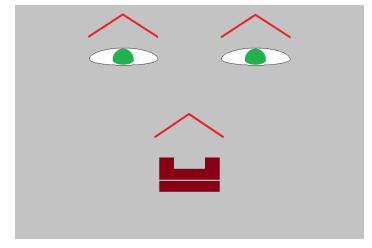

(a) Happiness

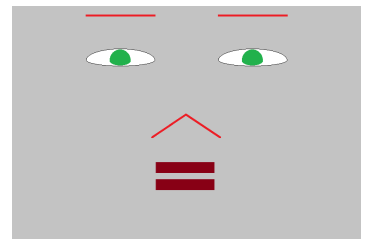

(c) Surprise

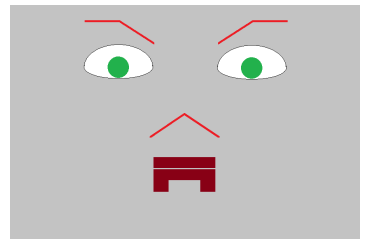

(b) Sadness

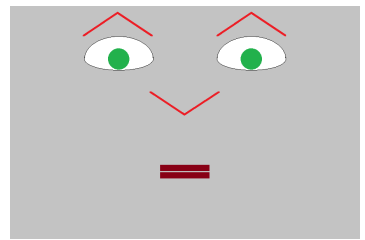

(d) Anger
Figure 9. Emotions represented by action units, according to Table 1.

Using PAD and SOR, intensity of emotion can be calculated through in Equation 1:

$$
\begin{aligned}
\Delta_{P} & =P_{\mathrm{n}_{\max }}-P(t) \\
\Delta_{A} & =A_{\mathrm{n}_{\max }}-A(t) \\
\Delta_{D} & =D_{\mathrm{n}_{\max }}-D(t) \\
I_{\mathrm{n}}(t) & =1-\left(K_{\mathrm{n}} \sqrt{\Delta_{P}{ }^{2}+\Delta_{A}{ }^{2}+\Delta_{D}}\right),
\end{aligned}
$$

where:

$I_{\mathbf{n}}(t)$ : Intensity of emotion along the time $t$;

$K_{\mathrm{n}}$ : Coefficient of emotion $n$;

$P_{\mathbf{n}_{\text {max }}}, A_{\mathbf{n}_{\text {max }}}, D_{\mathbf{n}_{\text {max }}}$ : Max values of pleasure, arousal and dominance, respectively;

$P(t), A(t), D(t)$ : Values of pleasure, arousal and dominance, respectively, according to SOR and PAD methods.

\subsection{Emotions and proxemics}

A finite state machine model will also be used to control the robot behavior. This model (Figure 10) has the behaviors, and therefore, the facial expressions the robot should make while interacting with the children, considering the child distance from the robot and if he/she is approaching or distancing.

In this finite state machine, three distance zones are considered: public, social and personal. According to the zone, the robot has a specific behavior. For the public zone the robot "sleeps", while in the other zones it stays awaken. During all time the robot measures the distance between children and it to determine which kind of the interaction it will make. Once in the public zone, the robot can wake up if it detects the child closer than $80 \mathrm{~cm}$. After waking 


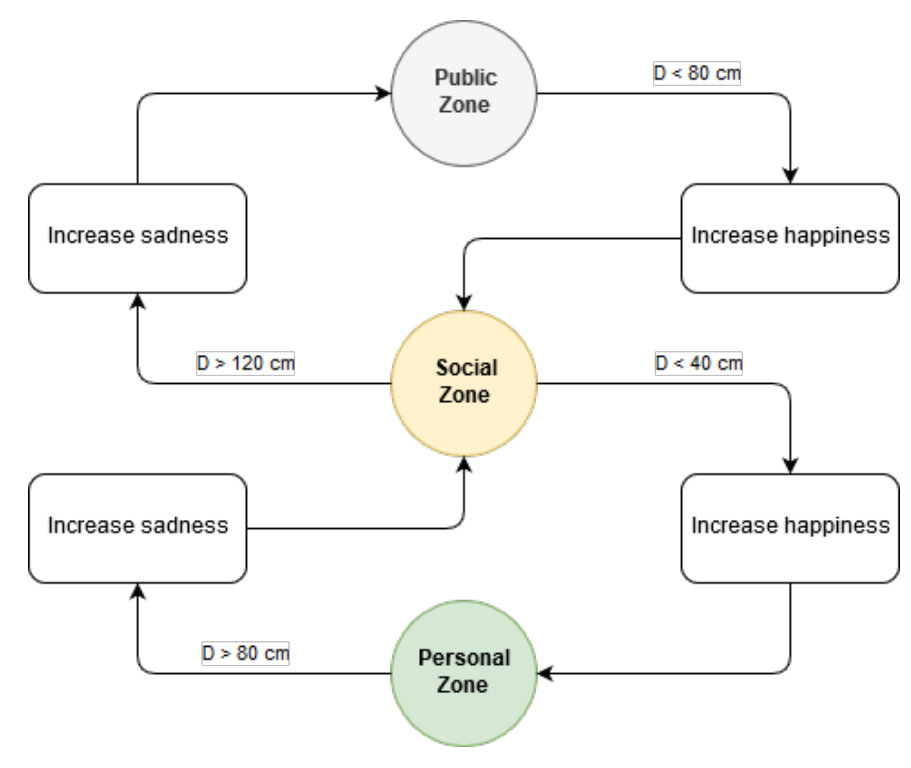

Figure 10. Finite state machine model of the robot emotional behavior according to proxemics. $D$ is the distance between robot and child.

up the robot spins towards the child (staying in front of the child, but without doing any linear movement). Otherwise, while the distance is larger than $80 \mathrm{~cm}$, the robot stays in public zone.

Once looking towards the child, the robot analyzes its distance from the child. If the child is between $40 \mathrm{~cm}$ and $80 \mathrm{~cm}$, it modulates the expression for a happy emotion. Then, it changes its state to the social zone. Another possible case is when the robot wakes up and the child is farther than $80 \mathrm{~cm}$ from the robot. In such case, it just comes back to sleep, keeping itself in the public zone.

In the social zone, the robot measures the distance again and if the child gets closer than $40 \mathrm{~cm}$, it gets happy and moves forward, changing to the personal zone. On the other hand, if the child stays between $40 \mathrm{~cm}$ and $120 \mathrm{~cm}$, it keeps in the social zone. In the case of the child distancing more than $120 \mathrm{~cm}$, the robot would understand the child wants less interaction, thus going to getting sad, going after to the public zone and sleeping.

In the case of the robot reaching the personal zone, it can stay in the personal zone if the distance is smaller than $80 \mathrm{~cm}$. Otherwise, it gets sad and moves backwards, as it understands the child would like a more distant interaction going to the social zone. All the information related to the finite state machine that guides the robot is better detailed in Figure 10.

\section{RESULTS}

Facial expressions are an important aspect to establish a non-verbal communication between humans. Therefore, the robot N-MARIA uses a tablet to show different expressions that represent emotions. Furthermore, the percentage of the emotion is also calculated, i.e., the intensity of the emotion the robot is displaying.

This can lead to a variety of facial expressions, since the robot can express not only the emotion itself, but its intensity. Additionally, an animation is made for the transition among emotion intensities.

Proxemics is intrinsically related to the emotion to be displayed, since it is one of the parameters that can be used to major child-robot interaction evolution. Thus, the percentage of emotions displayed are related to the proxemics. If the child is moving away from the robot, the expression displayed by the robot is sad or other negative one, while if the child approximates, the robot displays a positive emotion. The intensity is determined by the approaching or distancing of the children. Following this idea, this system brings a more natural and smooth animation of the affective display. Therefore, the expected result to the experiments is a better child-robot interaction.

\section{DISCUSSION}

Some previous studies, such as Santos et al. (2017), use programmed animation with a predefined sequence of images to make the animation, without representing intensity or modulating each part of the face separately. In this paper, instead, an algorithm-based dynamic face with more natural expressions is proposed, which varies its intensity according to the emotion that should be displayed to interact better with the children. Furthermore, an animation uses time equations, shown in Section 2.3, to consider the emotion decays or increases throughout the time.

Nevertheless, controlling the action units separately allows a better control of the expressions and creating several different ones, with graduated intensities and transitions. Thus, the robot facial expression can be better manipulated and more dynamic faces can be generated.

\section{CONCLUSION}

Robots have an increasing participation in our daily lives, aiding people in several areas, from industry to healthcare and therapies. This project is focused on the development of an assistive robot to work as a tool to enhance the therapy sessions of ASD children. As emotion perception and expression are major challenges for ASD children, alongside with socialization skills, the robot N-MARIA uses different expressions with animations to help these children in the development of these skills.

Furthermore, its facial expressions can give to the children a better experience, working as a tool, in their therapy. The animated expressions are expected to allow a better interaction, since the facial transitions are smoother and more natural.

\subsection{Future Implementations}

As future implementations the facial expressions of the robot will be able to move smoothly between two different emotions and not only inside the same emotion. In other words, it can, for example, decrease its sadness turning it, increasingly, into happiness. Currently, the facial expressions are animated only inside the same emotion.

These emotions are a way to respond to the child's reaction to the robot, thus favoring a good interaction. Having them 
animated smoothly can help a more natural child-robot interaction.

A set of experiments will be carried out with ASD children using the robot with this new facial display system. The experiments will consider the finite state machine shown in Figure 10 and, therefore, all situations evolved will be evaluated. In other words, the experiment will have the support of a mediator, who will conduct the test in two parts. In the first, the child will interact with full support of the mediator. The goal of this first part is to achieve all possible states of the finite state machine and evaluate the system. The second part will consist of a free interaction (supported by the mediator), evaluating the children's behavior towards the robot, using this new system of facial expression display.

\section{ACKNOWLEDGMENTS}

Authors would like to acknowledge the financial support from the Fundação de Amparo à Pesquisa do Espírito Santo (FAPES) and the technical support from the Federal University of Espirito Santo (UFES).

\section{REFERENCES}

Bakker, I., van der Voordt, T., Vink, P., and de Boon, J. (2014). Pleasure, arousal, dominance: Mehrabian and russell revisited. Current Psychology, 33(3), 405-421.

Binotte, V.P. (2018). Desenvolvimento de um robô socialmente assistivo com controle baseado em comportamento de seleção de ação para interação com crianças com TEA. Master's thesis, Universidade Federal do Espírito Santo, Vitória, ES.

Copeland, J.N. (2018). What is autism spectrum disorder? https://www.psychiatry.org/patients-families/ autism/what-is-autism-spectrum-disorder. Accessed on 2019-04-09.

Freitas-Magalhães, A. (2018). Facial Action Coding System 3.0-Manual de Codificação Científica da Face Humana-FM BabyFACS. Leya.

Friesen, E. and Ekman, P. (1978). Facial action coding system: a technique for the measurement of facial movement. Palo Alto, 3.

Harper, R.G., Wiens, A.N., and Matarazzo, J.D. (1978). Nonverbal communication: The state of the art. John Wiley \& Sons.

Hjortsjö, C.H. (1969). Man's face and mimic language. Studen litteratur.

Lafrance, M. (1979). Nonverbal behavior and communication-siegman, aw, feldstein, s.

Lambert, D. (2004). Body language. HarperCollins.

Mehrabian, A. and Russell, J.A. (1974). An approach to environmental psychology. the MIT Press.

Noldus Information Technology (2018). Facial Action Coding System (FACS). https://www.noldus.com/ facereader/facial-action-units. Accessed on 201905-03.

Rios-Martinez, J., Spalanzani, A., and Laugier, C. (2015). From Proxemics Theory to Socially-Aware Navigation: A Survey. International Journal of Social Robotics, 7(2), 137-153. doi:10.1007/s12369-014-0251-1. URL https://doi.org/10.1007/s12369-014-0251-1.
Santos, A., Goulart, C., Binotte, V., Rivera, H., Valadão, C., and Bastos, T. (2017). Development of an Artificial Dynamic Face Applied To an. In XIII Simpósio Brasileiro de Automação Inteligente, 448-452. Porto Alegre.

The National Institute of Mental Health (2018). Autism spectrum disorder. https://www.nimh.nih.gov/ health/topics/autism-spectrum-disorders-asd/ index.shtml. Accessed on 2019-04-09. 